\title{
Comparison of Plateletic Reactivity Affects Between Ticagrelor and Clopidogrel in Acute Myocardial Infarction Patients which Drive Coroner Intervention
}

\author{
Elly Usman ${ }^{1}$, Muhammad Fadil $^{2}$, Gestina Aliska ${ }^{3}$, Firdawati ${ }^{4}$ \\ \{ellyusman@med.unand.ac.id ${ }^{1}$ \}
}

\author{
Department of Pharmacology, Faculty of Medicine, Universitas Andalas Padang, Indonesia ${ }^{1}$ \\ Department of Cardiology and Vascular Medicine, Faculty of Medicine, Universitas Andalas Padang, \\ Indonesia $^{2}$ \\ Department of Community Health, University Kebangsaan Malaysia, Kuala Lumpur, Malaysia ${ }^{3}$
}

\begin{abstract}
This study aimed to determine the effect of P2Y12 inhibition by ticagrelor and clopidogrel and its effect on Major Adverse Cardiovascular Events (MACE) of patients with Acute Myocardial Infarction who underwent Percutaneous Coronary Intervention at Dr. M. Djamil General Hospital Padang. The design in this study was a quasi-experimental. The study was conducted at Dr. M. Djamil General Hospital Padang and the Department of Pharmacology and Therapeutics Faculty of Medicine Universitas Andalas in August December 2016. The population in this study were acute coronary syndrome patients who were treated in the Department of Cardiology of Dr. M. Djamil General Hospital Padang and underwent Percutaneous Coronary Intervention. The sample in this study were 32 samples with details of 16 samples for each group given platelets between ticagrelor and clopidogrel $(n=1)$ in patients with acute myocardial infarction who underwent percutaneous coronary intervention. Examination of platelet inhibition activity was carried out using the VerifyNow® P2Y12 assay (Accumetrics, San Diego, CA, USA) and conducted at the Central Laboratory of Dr. M. Djamil General Hospital Padang. VerifyNow ${ }^{\circledR}$ tests adenosine diphosphate-induced platelet function and is reported in the P2Y12 Reactions Unit (PRU) unit. The relationship between the Platelet Reaction Unit (PRU) value above or below the cut-off with the occurrence of MACE through the chisquare test. A two-tailed P-value of $<0.05$ was considered statistically significant. Data analysis was carried out in Stata version 14.2 (Stata Corporation). The results showed that administration of antiplatelet with clopidogrel obtained non-responsive platelet reaction unit (PRU) of $33.3 \%$ and hyperresponsiveness of $66.7 \%$ whereas in non-responsive ticagrelol found non-responsive PRU and experienced 100\% hyperresponsiveness. PRU with clopidogrel $128.1 \pm 99.6$ and ticagrelol $21.6 \pm 34.59$. Statistical test results obtained $\mathrm{p}$-value $=0.001(\mathrm{p}$-value $<0.05)$ it can be concluded that there are differences in PRU with clopidogrel and ticagrelol in patients with Acute Myocardial Infarction. There are differences in PRU with clopidogrel and ticagrelol in patients with Acute Myocardial Infarction.
\end{abstract}

Keywords: Clopidogrel, Acute Myocardial Infarction, Ticagrelor. 


\section{Introduction}

Coronary heart disease (CHD) is still one of the biggest problems in health problems. WHO states that ischemic heart disease causes more than 7 million $(12.8 \%)$ deaths worldwide. Data from the United States (US), CHD is the cause of one in every 6 deaths in 2009, based on data from the American Heart Association (AHA). The percentage of CHD patients in the US who died before receiving treatment was $73 \%$ in 2009 [1],[2].

Dual antiplatelet therapy with aspirin and clopidogrel has been the main pillar in the management of coronary heart disease, especially Acute Myocardial Infarction (AMI) and patients undergoing Percutaneous Coronary Intervention (PCI), with its role especially in the prevention of stent thrombosis after PCI. But, the recurrence rate for ischemic events remains high. The recurrence rate of ischemic events within 1 year after AMI in patients receiving combination therapy with aspirin and clopidogrel reached $10 \%$, with the incidence of stent thrombosis reaching $2 \%$. This residual risk is thought to be partly due to suboptimal platelet inhibition due to inadequate response to clopidogrel. Pharmacodynamic and pharmacokinetic studies of clopidogrel have found interindividual variations in the antiplatelet response of clopidogrel and an increased risk of ischemic and hyporesponder. This is thought to cause high platelet reactivity (HRPR) or high on-treatment platelet reactivity (HTPR). HPR is defined as the high rate of platelet reactivity several hours after obtaining a loading dose from an antiplatelet agent. The higher the residual platelet reactivity the higher the risk of cardiovascular events. Several studies have found a correlation between gene polymorphisms and active metabolite variability and clinical efficacy of clopidogrel [3],[4].

The importance of clopidogrel's role in AMI and PCI management, many parties are trying to find out how to integrate the data that has been obtained about patient management. These efforts are carried out to identify patients at risk of developing cardiovascular events about clopidogrel, obtain a more accurate estimate of the prognosis and adjustments of patient management based on the personal needs of each [5].

To overcome this problem, examinations to assess an individual's response to antiplatelet are being studied. These examinations, which aim to assess platelet inhibitory function and platelet reactivity, aim to identify individuals who are non-responders and adjust patient management based on individual circumstances. Several strategies have been tested in the management of individuals with non-responders to clopidogrel, including adjusting clopidogrel loading doses, using the newer generation prodrug thienopyridine groups such as prasugrel, or using new P2Y12 antagonists such as ticagrelor, in patients suspected of being hyporesponse to clopidogrel. Ticagrelor works directly as P2Y12 adenosine diphosphate receptor antagonists and does not require metabolic activation, so it is less affected by genetic polymorphisms and certain drugs such as proton pump inhibitors. Ticagrelor is considered to provide P2Y12 inhibitory effects that are faster, stronger, and more consistent than clopidogrel. However, the effect of ticagrelor compared to clopidogrel in its function for P2Y12 inhibition and comparison of the efficacy and safety of the two drugs, especially in Indonesia, is still not widely performed $[4],[6],[7]$.

\section{Methods}

\subsection{Study design, research sample}


The design in this study is a quasi-experimental. The study was conducted at Dr. M. Djamil General Hospital Padang and the Department of Pharmacology and Therapeutics Faculty of Medicine Universitas Andalas in August - December 2016. The population in this study were acute coronary syndrome patients who were treated in the Cardiology Department Dr. M. Djamil General Hospital and underwent Percutaneous Coronary Intervention. The sample criteria in this study were 32 samples with 16 samples for each group given platelets between ticagrelor and clopidogrel in patients with acute myocardial infarction who underwent percutaneous coronary intervention. The inclusion criteria of the study sample were patients with a diagnosis of acute myocardial infarction, male, nonpregnant woman, age $\geq 18$ years or $\leq 65$ years, pregnant woman, no liver function impairment, creatinine clearance $<30 \mathrm{ml} / \mathrm{min}$, a patient receiving clopidogrel therapy or ticagrelor and undergo percutaneous coronary intervention therapy. Exclusion criteria were patients who were on treatment using clopidogrel or ticagrelor and getting clopidogrel or ticagrelor therapy within 5 days before entering the study.

\subsection{Examination of platelet reactivity}

Examination of platelet inhibition activity was carried out using the VerifyNow ${ }^{\circledR}$ P2Y12 assay (Accumetrics, San Diego, CA, USA) and conducted at the Central Laboratory of Dr. M. Djamil General Hospital. VerifyNow ${ }^{\circledR}$ tests adenosine diphosphate-induced platelet function and is reported in the P2Y12 Reactions Unit. Patients are categorized as HRP or nonresponsive if the examination value is $\geq 208 \mathrm{PRU}$, and overresponsive if the examination value is $<90$ PRU.

\subsection{Operational definition}

Acute myocardial infarction is a diagnosis of acute myocardial infarction established by finding an increase and/or decrease in the value of cardiac markers, with at least 1 examination exceeding the 99th percentile of the upper limit of reference and at least one of the ischemic symptoms, ST-T segment changes or left branch file block new or suspected new 12-foot ECG examination, appearance of pathological Q waves on an electrocardiogram (EKG), evidence of new viable or suspected myocardial loss based on imaging and intracoronary thrombus found during angiography or autopsy [3],[8],[9].

Platelet reactivity is an examination of platelet inhibition activity using the VerifyNow® P2Y12 assay. Patients are categorized as HRP or nonresponsive if the examination value is $\geq$ 208 PRU, and overresponsive if the examination value is <90 PRU.

Major Adverse Cardiovascular Events (MACE) is a combination of cardiovascular death, nonfatal myocardial infarction, and nonfatal ischemic stroke. For this report, efficacy results including MACE are deaths that occur during patients treated at Dr.M. Djamil General Hospital.

\subsection{Data collection technique}

Data were collected from medical records of all outpatients and inpatients of the Cardiology and Vascular Medicine Division of Dr. M Djamil General Hospital who received clopidogrel and ticagrelor therapy who underwent PCI. From the medical records of the patients, data were collected including demographic characteristics/ data (name, age, gender, weight, a medical record number, CHD risk factors), history results, physical examination results, results of initial laboratory examination, diagnosis, and treatment of patients. The data above is used for selecting research subjects. Data regarding PRU values were obtained by examinations conducted at the Laboratory of Dr. M. Djamil General Hospital Padang. Examination of platelet 
reactivity is carried out using a patient's blood sample. Blood samples were taken in patients who had used clopidogrel and ticagrelor loading dose and performed PCI action. Blood samples are taken at 6-12 hours after loading dose and 30 days after PCI action. Blood samples are immediately checked using the VerifyNow ${ }^{\circledR}$ tool within 4 hours of taking blood.

\subsection{Data analysis}

Basic characteristics data will be presented with descriptive statistics. The relationship between PRU values above or below the cut-off with the occurrence of MACE through the chi-square test. The results are considered meaningful if the value of $\mathrm{P}<0.05$. Quantitative data that has been collected is processed and analyzed using the standard version 14.2 program.

\section{Results}

Characteristics of subjects (Table 1).

Table 1. Characteristics of research subjects

\begin{tabular}{ll}
\hline Parameter & $\mathrm{f}(\%) /$ mean \pm SD \\
\hline Age (years) & $56,38 \pm 7,44$ \\
Sex (male) & $29(90,6 \%)$ \\
Diagnosis & \\
IMA-EST & $23(71,9 \%)$ \\
IMA-NEST & $9(28,1 \%)$ \\
Risk factors & $17(53,1 \%)$ \\
Hypertension & $10(31,3 \%)$ \\
Diabetes mellitus & $25(78,1 \%)$ \\
Smoking & $16(50 \%)$ \\
Dyslipidemia & $19(59,4 \%)$ \\
Obesity & $3(9,4 \%)$ \\
Family history & $3(9,4 \%)$ \\
Menopause &
\end{tabular}

Table 1 of the known risk factors in the clopidogrel and ticagrelor groups was found to be older in the ticagrelor group compared to the clopidogrel group, as well as a higher diagnosis of IMAEST than the diagnosis of IMA-NEST in the clopidogrel group compared with ticagrelor. As for other risk factors, there were no differences in other cardiovascular risk factors between clopidogrel and ticagrelor groups. The difference between clopidogrel and ticagrelor groups (Table 2). 
Table 2. Characteristics of risk factors for clopidogrel and ticagrelor groups

\begin{tabular}{llll}
\hline Parameter & Clopidogrel & Ticagrelor & p-value \\
\hline Age (years) & $53,53 \pm 6,52$ & $58,88 \pm 7,46$ & $0,040^{*}$ \\
Sex (male) & $15(100 \%)$ & $14(82,4 \%)$ & 0,229 \\
Diagnosis & & & \\
IMA-EST & $14(93,3 \%)$ & $9(52,9 \%)$ & $0,014^{*}$ \\
IMA-NEST & $1(6,7 \%)$ & $8(47,1 \%)$ & \\
Risk factors & $9(60,0 \%)$ & $8(47,1 \%)$ & 0,464 \\
Hypertension & $5(33,3 \%)$ & $5(29,4 \%)$ & 0,811 \\
Diabetes mellitus & $12(80,0 \%)$ & $13(76,5 \%)$ & 1.000 \\
Smoking & $7(46,7 \%)$ & $9(52,9 \%)$ & 0,723 \\
Dyslipidemia & $10(66,7 \%)$ & $9(52,9 \%)$ & 0,430 \\
Obesity & $2(13,3 \%)$ & $1(5,9 \%)$ & 0,589 \\
Family history & $0(0 \%)$ & $3(17,6 \%)$ & 0,229 \\
Menopause & & & \\
\hline
\end{tabular}

$* \mathrm{P}<0.05$ significant

Table 2 shows that there were differences in age, diagnosis in the clopidogrel and ticagrelor groups ( $\mathrm{p}$-value $<0.05$ ). There were no sex differences, risk factors for hypertension, diabetes mellitus, smokers, dyslipidemia, obesity, family history and menopause in the clopidogrel and ticagrelor groups (p value> 0.05). PRU images in AMI patients (Figure 1).

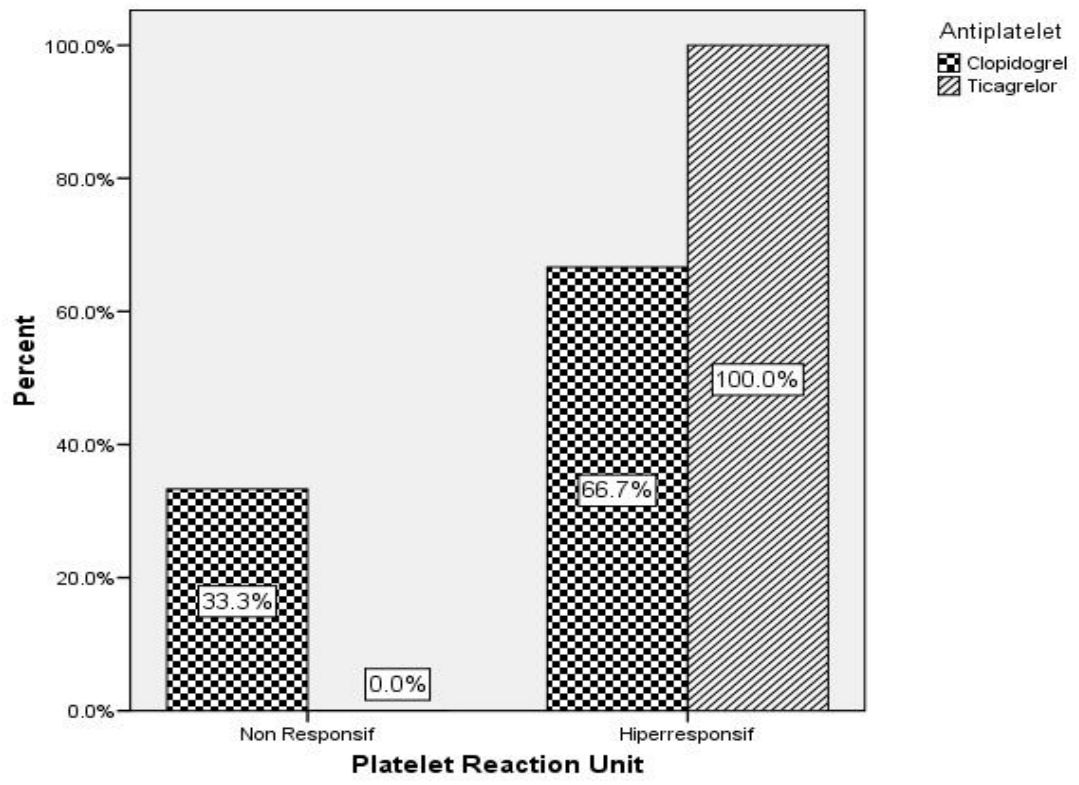

Fig. 1. Overview of Platelet Reaction Unit (PRU) in patients with Acute Myocardial Infarction (AMI) 
Figure 1 shows that the administration of antiplatelet with clopidogrel obtained non-responsive platelet reaction unit (PRU) of $33.3 \%$ and hyperresponsiveness of $66.7 \%$ whereas in nonresponsive ticagrelol found non-responsive PRU and experienced hyperresponsiveness of $100 \%$.

Table 3. Differences in Platelet Reaction Unit (PRU) with Clopidogrel and Ticagrelol in patients with Acute Myocardial Infarction (AMI)

\begin{tabular}{lllll}
\hline Antiplatelet & $\mathrm{n}$ & Mean $\pm \mathrm{SD}$ & $95 \%$ CI & $\mathrm{p}$-value \\
\hline Clopidogrel & 16 & $128,1 \pm 99,6$ & $49,4-163,6$ & 0,001 \\
Ticagrelol & 16 & $21,6 \pm 34,59$ & & \\
\hline
\end{tabular}

Table 3 shows the platelet reaction unit (PRU) with clopidogrel which is $128.1 \pm 99.6$ and ticagrelol is $21.6 \pm 34.59$. Statistical test results obtained $p=0.001(p<0.05)$ it can be concluded that there are platelet reaction unit differences with clopidogrel and ticagrelol in patients with Acute Myocardial Infarction (AMI).

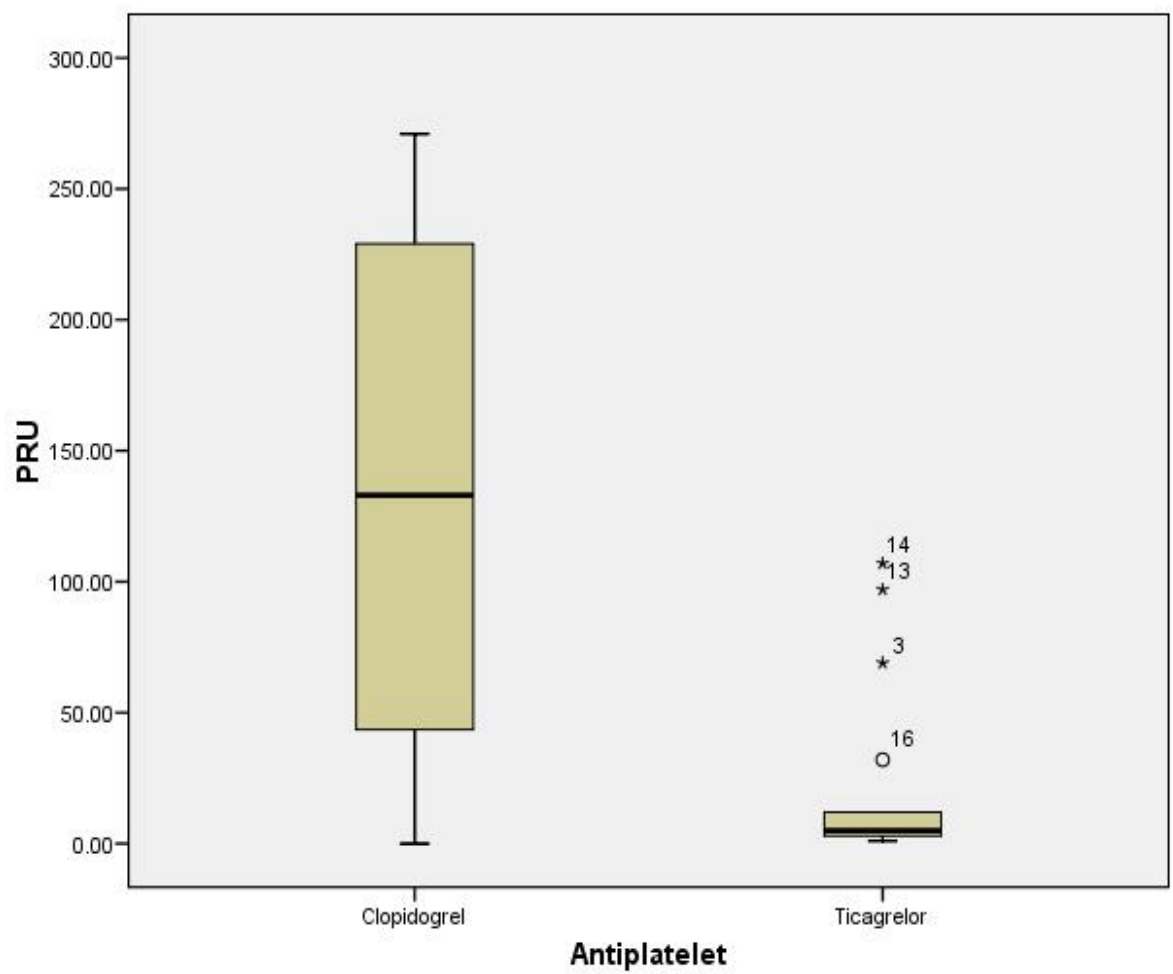

Fig. 2. Box Plot of the Platelet Reaction Unit (PRU) Plot for patients with Acute Myocardial Infarction (AMI) 


\section{Discussion}

In this study, there were no major cardiovascular events in both anti-platelet groups either clopidogrel or ticagrelor. Based on data on the basic characteristics and risk factors of subjects in this study it was found that AMI was found in many men, as well as the large proportion of patients with smoking, obesity, hypertension, and dyslipidemia.

Cigarettes, obesity, hypertension, and dyslipidemia are traditional cardiovascular risk factors. Smoking is associated with an increase in deaths from coronary heart disease through the mechanism of vascular endothelial injury due to cigarette smoke and a higher increase in myocardial oxygen demand in someone who smokes. Obesity is associated with an increased risk of hypertension, more blood vessel stiffness and a tendency to accumulate fat in the vascular endothelium which is all at risk for coronary heart disease. Hypertension is an independent risk factor for coronary heart disease that is associated with the incidence of vascular endothelial injury resulting in atherosclerotic plaque. While dyslipidemia, with the presence of endothelial injury that has occurred in the blood vessel wall, will accelerate the accumulation of fat in the lumen of blood vessels and result in atherosclerotic plaque

The study found that patients who received clopidogrel therapy tended to be younger and had a greater diagnosis of IMA-EST compared to the group of patients who received ticagrelor. The choice of antiplatelet therapy in this study was discretion from the physician in charge of the patient, so the absence of clear randomization of the choice of antiplatelet therapy might result in differences in the basic characteristics between patients who received clopidogrel compared with those who received ticagrelor. Differences in these basic characteristics may result in differences in major cardiovascular events between two anti-platelet groups.

In this study, it was found that the PRU levels of patients who received ticagrelor were all at the responsive level, while in the clopidogrel group 5 people were at the hyporesponsive level. Clopidogrel is a second generation thienopyridine in the form of prodrug and requires complex enteric and metabolism by cytochrome P450 (CYP) enzymes in the liver. In addition, the change of clopidogrel becomes active metabolically through a two-step process, namely the formation of 2-oxo-clopidogrel with the main role in the enzyme CYP2C19. The presence of the polymorphism of the CYP gene and the long process that clopidogrel passes as a prodrug to become active causes the hyporesponse to occur in PRU measurements.

Ticagrelor is a class of oral $\mathrm{P} 2 \mathrm{Y}$ receptor antagonists that reversibly inhibits platelets, which do not require liver bioactivation because they are active drugs. Antiplatelet ticagrelor activity is obtained through extracellular structural changes with allosteric modulation so that it binds to receptors independently of ADP and inhibits changes in conformation. This class of drugs ensures that anti-platelet activity is relatively more stable and inhibition of platelet reactivity is better than other drugs. In this study found a normal anti-platelet response in ticagrelor and the presence of hyporesponse to clopidogrel.

In this study, there were no major cardiovascular events within 30 days after treatment. The short period of monitoring after myocardial infarction and the relatively small number of samples made the absence of major cardiovascular events observed within 30 days after treatment.

Limitations of this study are the short period of monitoring after myocardial infarction because major cardiovascular events after myocardial infarction often occur within 1 year after the occurrence of myocardial infarction so that no major cardiovascular events can be detected within 30 days of monitoring. 
Acknowledgments. We would like to thank the Faculty of Medicine, Universitas Andalas for funding this research project and special thank to all respondents who participated in this study.

\section{References}

[1] Go A, Mozaffarian D, Roger V, Benjamin E, Berry J, Borden W, et al. Heart disease and stroke statistics--2013 update : a report from the American Heart Association. Circulation. 2013;127:e6245.

[2] Ng S, Soerianata S, Andriantoro H, Ottervanger JP, Grobbee DE. Timing of coronary collateral appearance during ST-elevation myocardial infarction. Interv Cardiol. 2012;4(1):137-43.

[3] Roffi M, Patrono C, Collet J-P, Mueller C, Valgimigli M, Andreotti F, et al. 2015 ESC Guidelines for the management of acute coronary syndromes in patients presenting without persistent STsegment elevation. Eur Heart J [Internet]. 2015;32(23):2999-3054.

[4] Garabedian T, Alam S. High residual platelet reactivity on clopidogrel: its significance and therapeutic challenges overcoming clopidogrel resistance. Cardiovasc Diagn Ther. 2013;3(1):2337.

[5] Krishna V, Diamond GA, Kaul S. The role of platelet reactivity and genotype testing in the prevention of atherothrombotic cardiovascular events remains unproven. Circulation. 2012;125(10):1288-303.

[6] Wallentin L, Becker RC, Budaj A, Cannon CP, Emanuelsson H, Held C, et al. Ticagrelor versus Clopidogrel in Patients with Acute Coronary Syndromes. N Engl J Med. 2009;361:1045-57.

[7] Storey RF, Angiolillo DJ, Patil SB, Desai B, Ecob R, Husted S, et al. Inhibitory effects of ticagrelor compared with clopidogrel on platelet function in patients with acute coronary syndromes: The PLATO (PLATelet inhibition and patient Outcomes) PLATELET substudy. J Am Coll Cardiol. Elsevier Inc.; 2010;56(18):1456-62.

[8] Thygesen K, Alpert JS, Jaffe AS, Simoons ML, Chaitman BR, White HD, et al. Third universal definition of myocardial infarction. Eur Heart J. 2012;33(20):2551-67.

[9] PERKI. Pedoman tatalaksana sindrom koroner akut. Pedoman Tatalaksana Sindr Koroner Akut. $2015 ; 88$. 